\title{
Expression evolution in yeast genes of single-input modules is mainly due to changes in trans-acting factors
}

\author{
Daryi Wang, ${ }^{1,6}$ Huang-Mo Sung, ${ }^{2,6}$ Tzi-Yuan Wang, $^{2}$ Chih-Jen Huang, ${ }^{2}$ Peggy Yang, ${ }^{2}$ \\ Tiffany Chang, ${ }^{2}$ Yang-Chao Wang, ${ }^{2}$ Da-Lun Tseng, ${ }^{2}$ Jen-Pey Wu, ${ }^{3}$ Tso-Ching Lee, $^{3}$ \\ Ming-Che Shih, ${ }^{4}$ and Wen-Hsiung Li ${ }^{2,5,7}$ \\ ${ }^{1}$ Research Center for Biodiversity, Academia Sinica, Taipei 115, Taiwan; ${ }^{2}$ Genomics Research Center, Academia Sinica, Taipei \\ 115, Taiwan; ${ }^{3}$ Department of Medical Research, Taichung Veterans General Hospital, Taichung 407, Taiwan; ${ }^{4}$ Department \\ of Biological Sciences and Roy J. Carver Center for Comparative Genomics, University of lowa, lowa City, Iowa 52242, USA; \\ ${ }^{5}$ Department of Ecology and Evolution, University of Chicago, Chicago, Illinois 60637, USA
}

\begin{abstract}
Both cis- and trans-regulatory mutations contribute to gene expression divergence within and between species. To estimate their relative contributions, we examined two yeast strains, BY (a laboratory strain) and RM (a wild strain), for their gene-expression divergence by microarray. Using these data and published ChIP-chip data, we obtained a set of single-regulator-regulated genes that showed expression divergence between BY and RM. We randomly selected 50 of these genes for further study. We developed a step-by-step approach to assess the relative contributions of cisand trans-variations to expression divergence by using pyrosequencing to quantify the mRNA levels of the BY and RM alleles in the same culture (co-culture) and in hybrid diploids. Forty genes showed expression divergence between the two strains in co-culture, and pyrosequencing of the BY/RM hybrid diploids showed that $45 \%(18 / 40)$ can be attributed to differences in trans-acting factors alone, $17.5 \%(7 / 40)$ mainly to trans-variations, $20 \%(8 / 40)$ to both cis- and trans-acting factors, 7.5\% (3/40) mainly to cis-variations, and 10\% (4/40) to cis-acting factors alone. In addition, we replaced the BY promoter by the RM promoter in each of 10 BY genes that were found from our microarray data to have expression divergence between BY and RM, and in each case our quantitative PCR analysis revealed a cis effect of the promoter replacement on gene expression. In summary, our study suggests that trans-acting factors play the major role in expression evolution between yeast strains, but the role of cis variation is also important.
\end{abstract}

[Supplemental material is available online at www.genome.org.]

Functional variation of a gene among individuals in a species can be due to nucleotide differences in coding regions or in regulatory elements. To date, most studies of molecular evolution have examined changes in protein-coding sequences (see Li 1997). Recently, several reviews argued that mutations in transcriptional regulation may have been a major cause for phenotypic evolution (Carroll 2000; Purugganan 2000; Stern 2000; Tautz 2000; Wray et al. 2003). Some studies have shown that natural genetic variations are heritable and can cause significant differences in gene expression (Brem et al. 2002; Cowles et al. 2002; Rockman and Wray 2002; Schadt et al. 2003). However, evolutionary changes in transcriptional regulation are still poorly understood.

Gene expression changes can arise from cis- or transchanges, or both. Variation(s) in cis-regulatory elements can be defined as polymorphism(s) in functional motifs of the promoter region or the DNA element(s) located within the transcribed region of the gene and/or a short distance outside of the transcribed region that influences the gene-expression level or timing or the mRNA stability. On the other hand, trans-regulatory variations are changes that affect the timing, level, or activity of the

\footnotetext{
${ }^{6}$ These authors contributed equally to this work.

${ }^{7}$ Corresponding author.

E-mail whli@uchicago.edu; fax (773) 702-9740.

Article published online before print. Article and publication date are online at http://www.genome.org/cgi/doi/10.1101/gr.6328907.
}

transcription factors (TFs) or other regulators that control the expression of the target genes (Yvert et al. 2003; Wittkopp et al. 2004). Thus, the effect of cis-variation should be allele specific, whereas trans-variation can affect both alleles in a diploid.

The relative contributions of cis- and trans-regulatory variations to expression evolution remain controversial. Several studies have found cases where intraspecies divergence in gene expression arose from mutations in cis-regulatory elements, such as in the cases of Gpdh and Cyp6g1 (the cytochrome P450 family) in Drosophila melanogaster (Laurie-Ahlberg and Bewley 1983; Daborn et al. 2002), beta-glucuronidase in Mus domesticus (Bush and Paigen 1992), and prolactin in the tilapia, Oreochromis niloticus (Streelman and Kocher 2002). Furthermore, recent studies reported that both intra- and interspecies divergences in gene expression during development among D. melanogaster, Drosophila yakuba, and Drosophila simulans are mainly due to cisvariations (Rifkin et al. 2003; Osada et al. 2006) and that the variations in expression levels of 29 genes between D. melanogaster and $D$. simulans are mainly due to cis-regulatory changes (Wittkopp et al. 2004).

However, using linkage analysis to map the genetic changes responsible for the expression differences of 2294 genes between the yeast strains BY4716 (a laboratory strain) and RM11-1a (a wild strain), Brem et al. (2002) and Yvert et al. (2003) found that the expression divergence of 1716 genes ( 75\%) between these 
two strains seemed to be mainly due to changes in trans-acting factors. More recently, Ronald et al. (2005) found that $\sim 25 \%$ of the 5727 genes that they have tested contain local regulatory variation between these two strains. By measuring allele-specific expression for 77 of these local regulatory variation-containing genes in a hybrid diploid with quantitative real time PCR, they estimated that in $52 \%-78 \%$ of these genes, local regulatory variation acts in cis. However, in some special circumstances, local regulatory variation identified through linkage analysis actually conferred trans-regulatory effects (Ronald et al. 2005; Rockman and Kruglyak 2006). Combined results from these studies suggest that trans-variations play a major role in expression divergence in yeast, with contributions from cis-variations. This is a very interesting finding because it is in contrast to the conclusion of the studies in Drosophila (Rifkin et al. 2003; Wittkopp et al. 2004; Osada et al. 2006). However, the studies in both Drosophila and yeast have not attempted to identify the direct target genes of a regulator, except for the case of Amn1P (Brem et al. 2002; Ronald et al. 2005), and the regulatory pathways of these regulators have not been investigated in detail. Here, we used a different approach to study the relative contributions of cis- and transregulatory mutations to expression evolution in yeast.

First, we chose yeast single-input module (SIM) genes, each of which is putatively regulated by a single transcription factor (TF). Since a SIM gene is regulated by a single immediateupstream TF, the chance for a change to occur in its trans-acting factors would, on average, be smaller than that for a multiple input module (MIM) gene, because a MIM gene is regulated by more TFs, and thus, likely by more trans factors than a SIM gene. For this reason, we hypothesize that the role of trans factors is less important for SIM genes than for MIM genes. Moreover, it would be simpler to figure out the molecular changes that cause the observed expression change in a SIM gene than in a MIM gene. To identify SIM genes we used data obtained by the ChIP-chip technique that combines chromatin-immunoprecipitation (ChIP) with DNA microarrays (chip) to map the promoter and its corresponding TFs (Lee et al. 2002; Harbison et al. 2004). Note that the current ChIP-chip data cannot guarantee that each of these genes is a true SIM gene, so they should be taken as "putative" SIM genes. However, such a gene is unlikely to be regulated by many TFs.

Second, we used two different budding yeast strains BY4741 (denoted as BY) and RM11-1a (RM). These two strains proliferate rapidly and have propagated under different environmental conditions for decades. As a result, these two strains display substantial divergence in gene expression and are ideal for studying expression divergence within species.

Third, we took advantage of the fact that yeast can propagate as haploids or diploids and that the pyrosequencing technique can be used to estimate the relative expression levels of two alleles in an mRNA pool if there is nucleotide polymorphism in the coding region. We first used microarrays to identify and select SIM genes that showed expression differences between the BY and RM strains. Next, we used pyrosequencing to check the expression differences of each selected gene when the two strains were grown in separate cultures, and selected those genes that showed significantly different expression levels for further analysis. The expression differences could be due to a combination of differences in environmental factors such as glucose concentration and genetic (trans or cis) factors. Then, we grew the two strains in the same culture (co-culture) and used pyrosequencing to estimate the relative expression levels of the two alleles of a gene (one from each of the two strains). This co-culturing should remove the environmental effects.

Fourth, we formed a BY/RM hybrid diploid and used pyrosequencing to study the relative expression levels of the two alleles of a gene in the diploid (where the two alleles are from the two different strains). This procedure should remove the effects of trans factors, so the remaining differences, if any, should be due to the cis effects.

Finally, for some of the genes selected we have also swapped the promoters of the two strains to verify the cis effects.

In summary, the experimental procedure we used provided a rigorous means to separate the cis and trans effects. As will be seen, our conclusion is that trans factors play a major role for the expression divergence between the two yeast strains, even for SIM genes. However, it will become clear that cis effects are also common. Since we are still in the process of obtaining data for MIM genes, we cannot yet test our hypothesis that the role of trans factors is less important for SIM genes than for MIM genes.

\section{Results}

\section{SIM genes that show different expression levels between strains}

We used microarrays to compare gene expression profiles of BY and RM cells from exponentially growing cells. There were 1360 genes that showed significant $\left(P<0.5 \times 10^{-4}\right)$ expression differences between the two strains (Supplemental Table 1). We used a collection of Saccharomyces cerevisiae TFs and their target genes predicted from ChIP-chip data (Harbison et al. 2004). This database includes 203 known TFs and their downstream target genes, from which we collected 1049 genes, each of which is uniquely bound by only one of 72 TFs at a significance level of $P<10^{-3}$ (Harbison et al. 2004). By incorporating the single-input modules inferred from the ChIP-chip data (Lee et al. 2002; Harbison et al. 2004) with our expression data, we identified 219 putative single-regulator regulated (SIM) genes that showed different expression levels between the BY and RM strains (Supplemental Table 2).

\section{Allele quantification of SIM genes}

For pyrosequencing analyses, we randomly selected 50 SIM genes from a pool of 80 genes in Supplemental Table 2, for which the regulatory domain and DNA-binding domain of the upstream TFs are known (see Supplemental Table 3 for the gene list and DNA sequence comparison). An additional selection criterion used was that the gene should have polymorphism(s) in the transcribed region between $\mathrm{BY}$ and RM, which can be used as the target for pyrosequencing analysis.

We used pyrosequencing to check the expression differences in each selected gene between the BY and RM strains grown in separate cultures. We found that eight of the 50 selected genes showed similar expression levels, while the remaining 42 genes showed significantly different expression levels between the BY and RM strains (Supplemental Table 4). The observed expression differences in separate cultures could be due to a combination of environmental and genetic factors such as glucose concentration, trans factors, or cis factors. Next, we used pyrosequencing to quantify the relative expression levels of the RM/BY alleles of these 50 SIM genes when the strains are grown together in coculture. We found four of these 50 genes did not show significant expression difference in both separate cultures and co-culture;

\section{Genome Research}

www.genome.org 
these four genes were removed from further analysis. In addition, we found that six of the remaining 46 genes have similar allele expression levels under co-culture $(\mathrm{RM} / \mathrm{BY} \approx 1$; Supplemental Table 4), but show different expression levels when grown separately. Since this co-culturing system should remove the environmental effects, our results indicated that 13\% (6/46) of the expression divergence observed under separate cultures was mainly due to interactions between genetic factors and environmental factors. Interestingly, there were five genes that showed a similar expression level in separate cultures of the BY and RM strains, but showed significant expression difference in the BY and RM coculture system. The nonsignificant difference in expression level under separate cultures could be in part due to large standard errors (two of the five genes had particularly large standard errors) or due to stronger effects of environmental factors than genetic factors (Supplemental Table 4). When grown in separate cultures, the RM strain grows faster and consumes glucose at a faster rate, and this difference in glucose concentration may affect the expression of some genes.

To further characterize the effects of cis- and trans-variations on the 40 genes that showed significant expression differences between the BY and RM alleles in the co-culture system, and are therefore likely to be affected by the genetic variations between $\mathrm{BY}$ and $\mathrm{RM}$, we generated a $\mathrm{BYa} / \mathrm{RM} \alpha$ and a $\mathrm{RMa} / \mathrm{BY} \alpha$ hybrid diploid strain and examined the relative expression levels of the BY and RM alleles of the 40 genes in each strain by pyrosequencing (see Methods). Because the trans-acting effects should be the same in the same genetic background, an equal level of expression of the BY and RM alleles in a hybrid diploid indicates differences in trans-regulation. Therefore, if trans-regulatory divergence completely explains the expression difference of a given gene between the two strains, the allele-specific expression will be approximately the same in the hybrids. On the other hand, if the allele-specific expression of a given gene is different in the hybrid, the difference can be attributed to cis-regulatory divergence. If cis-regulatory divergence completely explains the expression difference of a given gene between the two strains, the allele-specific expression ratios in the hybrid and parental strains should be approximately equal $\left(\mathrm{RM}_{\text {parent }} / \mathrm{BY}_{\text {parent }}=\mathrm{RM}_{\text {hybrid }} /\right.$ $\mathrm{BY}_{\text {hybrid }}$ ). If the expression levels of the $\mathrm{BY}$ and $\mathrm{RM}$ alleles in a hybrid are different and the ratio $\mathrm{RM}_{\text {parent }} / \mathrm{BY}_{\text {parent }}$ is different from $\mathrm{RM}_{\text {hybrid }} / \mathrm{BY}_{\text {hybrid }}$, the expression difference between the BY and RM alleles should be due to a combination of cis- and transregulatory effects. Our results, which are summarized in Table 1, showed that $45 \%(18 / 40)$ of these 40 cases can be attributed to the differences in trans-acting factors alone, $17.5 \%$ (7/40) mainly to trans-variations, $20 \%(8 / 40)$ to both cis- and trans-acting factors, $7.5 \%(3 / 40)$ mainly to cis-variations, and $10 \%(4 / 40)$ to cisacting factors alone (Table 1 ).

In summary, variations in expression or sequence of transacting factors were mainly responsible for $62.5 \%$ of the expression difference observed between the BY and RM strains. In total, trans-acting factors affected $90 \%$ of the genes that showed expression differences between the BY and RM strains if the minor trans-effects were also included, while the corresponding value for cis-factors is only $55 \%$.

\section{Confirmation of the cis-regulatory effect by promoter swapping}

We performed promoter-swapping experiments to confirm the cis-effects inferred by the SIM gene DNA sequence comparisons and microarray data. The candidate genes for promoter swapping were selected by three criteria: (1) the expression is significantly different between the BY and RM strains, (2) the expression of the corresponding upstream TF is similar for the BY and RM strains, and (3) the binding domain and activation domain of the TF have no nonsynonymous mutation between the BY and RM strains. Thus, these genes were selected to minimize trans effects and enhance cis effects. For each gene selected, there were SNPs identified in the intergenic region (which usually includes the promoter region) between the BY and RM strains, though the SNPs did not fall into the predicted TF-binding site (Fig. 1). The expression regulation of SIM genes appears to be more complicated than we expected, so we examined whether the cisvariation affected the gene expression after the intergenic region of the BY allele was replaced with the intergenic region of the RM allele. Seventeen genes with a significant difference between the $\mathrm{BY}$ and RM strains in expression level in the array data $\left(P<1.0 \times 10^{-4}\right)$ were selected for promoter-region swapping, and we succeeded in 10 cases; we did not have successful transformants for the remaining seven genes. The sequence comparisons of the promoter regions of these 10 genes are shown in Figure 1 . There are 33, 24, 6, 6, 6, 5, 5, 5, 9, and 6 nucleotide differences in the promoter regions of YCR075C, YDL137W, YEL052W, YEL053C, YFL004W, YGR157W, YLR438W, YML075C, YOR306C, and YPL157W between BY and RM. After the replacement of the BY promoter sequence (300-800 bp) with the RM sequence, the expression of the gene was determined by quantitative real time PCR (qRT-PCR).

The relative expression levels of RM and BY (RM/BY, Column 4) and of the Swap strain and BY (Swap/BY, column 6) determined by qRT-PCR are summarized in Table 2. (Some of the specific promoter swapping had several different transformants that were from different transformation events, and they showed the same phenotype and similar qRT-PCR results. The qRT-PCR results for four of the 10 genes were actually obtained from two transformants.) For comparison, the RM/BY expression ratios estimated from our microarray data and pyrosequencing data are also listed in Table 2 (column 2). Although the RM/BY expression ratios estimated from microarray, real-time PCR, and pyrosequencing data are not identical, they do show the same trend; e.g., if the RM/BY ratio from the microarray data was $<1$, it was also $<1$ from the real time PCR and pyrosequencing data (Table 2). Under the assumption of no effect of cis-variation on the expression level of a gene, the Swap/BY ratio is expected to be 1 . Therefore, our data indicate the presence of cis-effect in each of the 10 genes studied, because the Swap/BY ratio is different from 1 for all of the 10 genes (Table 2). For gene YDL137W, the estimated Swap/BY ratio is 1.45, which is similar to the estimate for the RM/BY ratio from qRT-PCR data (1.44, Table 2). This observation suggests that the BY allele with the promoter of the RM allele showed an expression level similar to that of the RM allele. In other words, the lower expression level of the RM allele was mainly due to its promoter region, so that the expression difference between the RM and BY alleles was mainly due to cis-effects. A similar comment applies to the gene YEL053C. However, for the remaining eight genes, the Swap/BY ratio was different from the RM/BY ratio in each case, so there was also a trans-effect in each case (Table 2). Therefore, for these eight genes, the expression divergence between $\mathrm{BY}$ and $\mathrm{RM}$ is due to variations in both promoter sequences and upstream trans-regulatory factors. This conclusion is largely consistent with the pyrosequencing data summarized in Table 1, though the data in Table 2 indicates a 
Table 1. Pyrosequencing results of the 40 genes that showed significant expression divergence between BY and RM

\begin{tabular}{|c|c|c|c|c|c|}
\hline ORF $^{a}$ & Separate culture & Co-culture & $\operatorname{BY}(a) \times \operatorname{RM}(\alpha)$ hybrid & $\operatorname{RM}(a) \times \operatorname{BY}(\alpha)$ hybrid & Effect \\
\hline YBL050W & $0.92 \pm 0.025$ & $0.66 \pm 0.057$ & $1.03 \pm 0.082$ & $0.99 \pm 0.050$ & trans alone \\
\hline YDL084W & $0.83 \pm 0.019$ & $0.86 \pm 0.033$ & $1.03 \pm 0.034$ & $1.04 \pm 0.038$ & trans alone \\
\hline YER103W & $0.59 \pm 0.063$ & $0.53 \pm 0.031$ & $1.05 \pm 0.048$ & $1.03 \pm 0.053$ & trans alone \\
\hline YGL198W & $0.95 \pm 0.019$ & $0.92 \pm 0.032$ & $1.02 \pm 0.033$ & $1.02 \pm 0.032$ & trans alone \\
\hline YGL248W & $0.84 \pm 0.066$ & $0.80 \pm 0.064$ & $1.06 \pm 0.031$ & $1.05 \pm 0.041$ & trans alone \\
\hline YGR241C & $1.07 \pm 0.062$ & $1.21 \pm 0.090$ & $1.00 \pm 0.014$ & $0.97 \pm 0.044$ & trans alone \\
\hline YHR146W & $0.78 \pm 0.030$ & $0.61 \pm 0.051$ & $0.95 \pm 0.026$ & $0.98 \pm 0.057$ & trans alone \\
\hline YJL130C & $1.01 \pm 0.045$ & $1.18 \pm 0.037$ & $1.00 \pm 0.051$ & $1.00 \pm 0.042$ & trans alone \\
\hline YJR077C & $2.14 \pm 0.151$ & $1.95 \pm 0.162$ & $1.00 \pm 0.034$ & $1.00 \pm 0.038$ & trans alone \\
\hline YKL072W & $0.49 \pm 0.092$ & $0.47 \pm 0.080$ & $1.04 \pm 0.051$ & $0.96 \pm 0.057$ & trans alone \\
\hline YKR054C & $0.66 \pm 0.098$ & $0.67 \pm 0.107$ & $0.99 \pm 0.076$ & $1.02 \pm 0.082$ & trans alone \\
\hline YLR223C & $0.85 \pm 0.031$ & $0.68 \pm 0.024$ & $0.99 \pm 0.039$ & $0.97 \pm 0.052$ & trans alone \\
\hline YNL094W & $0.69 \pm 0.044$ & $0.70 \pm 0.038$ & $1.00 \pm 0.045$ & $1.03 \pm 0.026$ & trans alone \\
\hline YNL113W & $1.12 \pm 0.039$ & $0.91 \pm 0.029$ & $1.00 \pm 0.040$ & $0.99 \pm 0.047$ & trans alone \\
\hline YNR050C & $0.88 \pm 0.029$ & $0.81 \pm 0.044$ & $1.00 \pm 0.044$ & $1.00 \pm 0.073$ & trans alone \\
\hline YOL021C & $1.02 \pm 0.020$ & $0.91 \pm 0.040$ & $1.00 \pm 0.092$ & $1.03 \pm 0.093$ & trans alone \\
\hline YOR326W & $0.83 \pm 0.064$ & $0.90 \pm 0.038$ & $0.97 \pm 0.026$ & $0.97 \pm 0.042$ & trans alone \\
\hline YPR154W & $0.80 \pm 0.046$ & $0.63 \pm 0.046$ & $0.98 \pm 0.037$ & $1.04 \pm 0.050$ & trans alone \\
\hline YAL037W & $0.54 \pm 0.032$ & $0.78 \pm 0.045$ & $1.09 \pm 0.036$ & $1.10 \pm 0.035$ & major trans effect \\
\hline YCR075C & $0.52 \pm 0.028$ & $0.62 \pm 0.041$ & $1.09 \pm 0.036$ & $1.06 \pm 0.025$ & major trans effect \\
\hline YDR232W & $1.64 \pm 0.120$ & $1.58 \pm 0.084$ & $1.17 \pm 0.050$ & $1.19 \pm 0.053$ & major trans effect \\
\hline YLR438W & $1.96 \pm 0.104$ & $2.70 \pm 0.192$ & $1.54 \pm 0.049$ & $1.57 \pm 0.054$ & major trans effect \\
\hline YML075C & $4.11 \pm 0.294$ & $3.77 \pm 0.449$ & $1.21 \pm 0.028$ & $1.25 \pm 0.050$ & major trans effect \\
\hline YOL144W & $0.69 \pm 0.057$ & $0.67 \pm 0.078$ & $1.19 \pm 0.031$ & $1.12 \pm 0.045$ & major trans effect \\
\hline YPL264C & $0.37 \pm 0.059$ & $0.56 \pm 0.080$ & $1.09 \pm 0.034$ & $1.05 \pm 0.021$ & major trans effect \\
\hline YFL016C & $0.83 \pm 0.077$ & $0.85 \pm 0.025$ & $0.83 \pm 0.035$ & $0.85 \pm 0.029$ & cis alone \\
\hline YFL037W & $0.97 \pm 0.044$ & $0.89 \pm 0.032$ & $0.87 \pm 0.033$ & $0.88 \pm 0.039$ & cis alone \\
\hline YGL200C & $1.05 \pm 0.015$ & $1.10 \pm 0.042$ & $1.10 \pm 0.025$ & $1.10 \pm 0.039$ & cis alone \\
\hline YIL117C & $0.61 \pm 0.056$ & $0.83 \pm 0.041$ & $0.87 \pm 0.046$ & $0.86 \pm 0.042$ & cis alone \\
\hline YDL124W & $0.35 \pm 0.083$ & $0.25 \pm 0.026$ & $0.40 \pm 0.037$ & $0.42 \pm 0.072$ & major cis effect \\
\hline YMR318C & $0.67 \pm 0.055$ & $0.71 \pm 0.021$ & $0.77 \pm 0.048$ & $0.84 \pm 0.040$ & major cis effect \\
\hline YOR306C & $0.46 \pm 0.043$ & $0.48 \pm 0.025$ & $0.55 \pm 0.031$ & $0.60 \pm 0.060$ & major cis effect \\
\hline YEL052W & $0.62 \pm 0.038$ & $0.70 \pm 0.065$ & $0.83 \pm 0.063$ & $0.86 \pm 0.038$ & both cis and trans \\
\hline YGR157W & $0.52 \pm 0.030$ & $0.57 \pm 0.040$ & $0.77 \pm 0.021$ & $0.80 \pm 0.042$ & both cis and trans \\
\hline YJR078W & $0.38 \pm 0.041$ & $0.28 \pm 0.066$ & $1.54 \pm 0.086$ & $1.60 \pm 0.065$ & both cis and trans \\
\hline YKL152C & $0.63 \pm 0.091$ & $0.64 \pm 0.095$ & $0.77 \pm 0.041$ & $0.78 \pm 0.035$ & both cis and trans \\
\hline YOL140W & $1.18 \pm 0.070$ & $1.28 \pm 0.033$ & $1.11 \pm 0.042$ & $1.12 \pm 0.041$ & both cis and trans \\
\hline YOR049C & $0.28 \pm 0.054$ & $0.28 \pm 0.066$ & $0.57 \pm 0.064$ & $0.64 \pm 0.080$ & both cis and trans \\
\hline YOR150W & $1.72 \pm 0.102$ & $1.43 \pm 0.155$ & $1.16 \pm 0.059$ & $1.24 \pm 0.057$ & both cis and trans \\
\hline YPL157W & $0.67 \pm 0.055$ & $0.63 \pm 0.041$ & $0.88 \pm 0.035$ & $0.83 \pm 0.049$ & both cis and trans \\
\hline
\end{tabular}

Data presented are the expression ratios of the RM/BY alleles in separate cultures, in co-culture, and in two BY $\times$ RM hybrids. Each reaction was repeated at least three times from different RNA and cDNA samples.

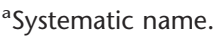

somewhat more important role of cis variation than inferred from the result in Table 1 . Note, however, that the 10 genes were selected for promoter replacement using the three criteria mentioned above. These criteria can lead to an overestimate of the contribution of cis elements to expression divergence.

\section{Relationship between sequence polymorphism and cis versus trans effects}

In comparing the DNA sequences of the two strains, we found that the polymorphism rate in the upstream intergenic regions of the genes whose expression divergence is affected mainly by cisvariation is higher than that in the upstream regions of the genes whose expression divergence was affected mainly by transvariation (0.0102 vs. 0.0051 nucleotide differences per site, $P<0.05)$. All of the genes whose expression level is affected mainly by cis-regulatory variations show polymorphisms in their promoter (upstream) regions and in their coding regions (Table 3 ); however, in the coding regions, the polymorphism rate in genes with cis-regulatory variation is not significantly different from that in the genes with trans-regulatory variation (0.0047 vs. 0.0028). Similarly, Ronald et al. (2005) observed a greater polymorphism rate $(0.0071)$ in the upstream regions of the 1233 genes showing self-linkage, compared with upstream regions of 3949 genes without self-linkage (0.0040); the increase was not restricted to the region most likely to contain regulatory elements, but extended for at least $1 \mathrm{~kb}$ upstream of the genes. They also observed that the polymorphism rate was also higher in the coding regions of genes showing self-linkage (0.0044 vs. 0.0029).

\section{Discussion}

Our allele quantification analysis with pyrosequencing showed that trans-acting factors affect most of the expression differences observed between the BY and RM strains, in accordance with the observation of Brem et al. (2002), Yvert et al. (2003), and Ronald et al. (2005). Indeed, our data suggested that $90 \%$ of genes with expression difference between BY and RM are affected by transacting regulatory factors, though half of them are also influenced by differences in cis-acting regulatory elements. Taken together, our results show that even for SIM genes, which likely have only one or few immediate upstream TFs, trans-regulatory variation plays a major role in expression divergence between the two yeast strains. Therefore, it is reasonable to speculate that transvariation is a major determinant in the genome-wide expression 
divergence in the yeast $S$. cerevisiae. Yvert et al. (2003) reported that 1716 of the 2294 genes that showed expression differences between BY and RM are not selflinked in the genome. The data suggested that $75 \%(1716 / 2294)$ of the genes are affected by trans-variation between BY and RM. Their results also suggested that most regulatory genetic variation does not reside in the TFs themselves, because most of the transvariation effect observed between BY and RM does not map to the immediate upstream TFs. In some special circumstances, local regulatory variation identified through linkage analysis actually confers trans-regulatory effects (Ronald et al. 2005; Rockman and Kruglyak 2006). Ronald et al. (2005) examined 77 genes that showed self-linkage for the presence of allele-specific expression (ASE) in a diploid hybrid of the two parent strains, BY and RM (cis-variance will cause allele-specific expression in a diploid hybrid). Their results showed that $57 \%$ (44) of the 77 assayed genes have ASE, suggesting that trans-acting local variation is likely to be responsible for a minority of the self-linkages tested. Overall, these data and our data suggest that trans-variation plays a major role in expression divergence in yeast.

It has been suggested that promoters may be more evolvable than coding regions (Gerhart and Kirschner 1997; Carroll et al. 2001). Even though our allele quantification with pyrosequencing showed that only $10 \%$ of SIM genes were affected by the variations in cisregulatory elements alone, the data also showed that $45 \%$ of SIM genes were affected by variations in both cis- and trans-regulatory elements. Indeed, the expression in promoter-swapped strains showed that the polymorphisms in the promoter region contributed to the expression divergence of the two yeast strains in each of the 10 genes studied (Table 2). On the other hand, a change in a regulator may affect the expression of multiple downstream genes. One example in our study is the HAP4 gene. The HAP4 mRNA level in the wild strain is $18 \%$ higher than the level in the laboratory strain, and it may be responsible or partially responsible for the expression difference of $42 \%(8 / 19)$ of its target genes between the BY and RM strains (our microarray data). HAP4 was suggested to act as a target of carbon-source regulation, because its mRNA level increased about 10-fold during nonfermentative conditions (Forsburg and Guarente 1989; DeRisi et al. 1997). Evolution of transacting factors may be related to the relaxation of selection pressure or adaptation to the rich medium growth condition in the laboratory strain (Gu et al. 2005).

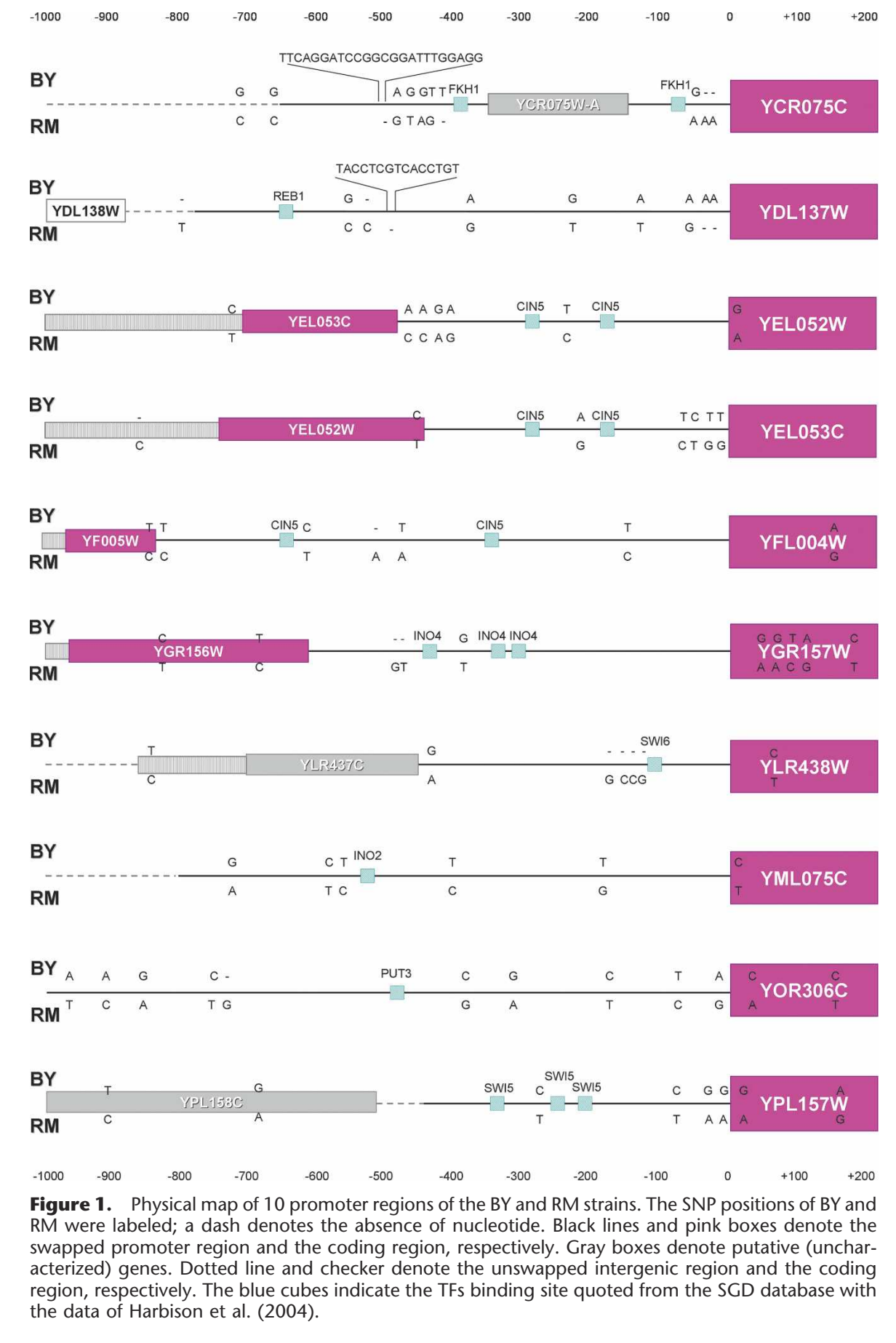

Figure 1. Physical map of 10 promoter regions of the BY and RM strains. The SNP positions of BY and RM were labeled; a dash denotes the absence of nucleotide. Black lines and pink boxes denote the swapped promoter region and the coding region, respectively. Gray boxes denote putative (uncharacterized) genes. Dotted line and checker denote the unswapped intergenic region and the coding region, respectively. The blue cubes indicate the TFs binding site quoted from the SGD database with the data of Harbison et al. (2004).

(tory strain (Gu et al. 2005).

Cis-regulatory variations are more readily studied at the molecular level compared with trans-regulatory variations. The effect of trans-regulatory variations is difficult to pursue at the molecular level because trans-regulatory variations could be due to differences in any combination of expression level, DNA-binding affinity, cofactor interaction, and post-translation modification of upstream factors.

The influence of changes in cis- and/or trans-regulatory elements during evolution is still a controversial issue. The conclusion can vary from study to study, depending on the organism studied (Brem et al. 2002; Schadt et al. 2003; Yvert et al. 2003; 
Table 2. Relative gene expression levels of the SIM genes between promoter-swapped strains and the BY4741 strain

\begin{tabular}{|c|c|c|c|c|c|c|c|}
\hline \multirow[b]{2}{*}{ SIM gene ${ }^{a}$} & \multicolumn{2}{|c|}{ Ratio RM/BY } & \multirow[b]{2}{*}{$\Delta \Delta \mathrm{Ct} \mathrm{RM} / \mathrm{BY}$} & \multirow[b]{2}{*}{ Ratio RM/BY $(95 \% \mathrm{Cl})$} & \multirow[b]{2}{*}{$\Delta \Delta$ Ct Swap/BY } & \multirow[b]{2}{*}{ Ratio Swap/BY $(95 \% \mathrm{Cl})$} & \multirow[b]{2}{*}{ Effect } \\
\hline & Arrays & Pyroseq. & & & & & \\
\hline YCR075C & 0.74 & 0.52 & $0.59 \pm 0.16$ & $0.67(0.53-0.81)^{\mathrm{b}}$ & $-0.11 \pm 0.09$ & $1.08(0.96-1.2)$ & major trans effect \\
\hline YDL137W & 1.27 & NA & $-0.52 \pm 0.10$ & $1.44(1.24-1.64)^{\mathrm{b}}$ & $-0.53 \pm 0.03$ & $1.45(1.39-1.51)^{c}$ & cis alone \\
\hline YEL052W & 0.83 & 0.62 & $1.04 \pm 0.18$ & $0.49(0.35-0.63)^{c}$ & $0.57 \pm 0.13$ & $0.67(0.55-0.79)^{c}$ & both cis and trans \\
\hline YEL053C & 0.84 & NA & $0.60 \pm 0.23$ & $0.67(0.45-0.89)^{b}$ & $0.64 \pm 0.12$ & $0.64(0.54-0.74)^{c}$ & cis alone \\
\hline YFL004W & 0.68 & NA & $1.00 \pm 0.23$ & $0.50(0.32-0.68)^{c}$ & $-0.40 \pm 0.17$ & $1.33(1.01-1.65)$ & both cis and trans \\
\hline YGR157W & 0.66 & 0.52 & $1.71 \pm 0.27$ & $0.31(0.19-0.43)^{c}$ & $0.92 \pm 0.32$ & $0.54(0.28-0.80)^{\mathrm{b}}$ & both cis and trans \\
\hline YLR438W & 1.31 & 2.70 & $-1.57 \pm 0.31$ & $3.02(1.74-4.3)^{\mathrm{b}}$ & $0.12 \pm 0.03$ & $0.92(0.88-0.96)^{c}$ & major trans effect \\
\hline YML075C & 1.97 & 3.77 & $-1.18 \pm 0.07$ & $2.27(2.05-2.49)^{c}$ & $-0.38 \pm 0.08$ & $1.30(1.14-1.46)^{\mathrm{b}}$ & major trans effect \\
\hline YOR306C & 0.58 & 0.46 & $1.42 \pm 0.21$ & $0.38(0.28-0.48)^{c}$ & $0.25 \pm 0.08$ & $0.84(0.74-0.94)^{b}$ & major trans effect \\
\hline YPL157W & 0.93 & 0.67 & $0.31 \pm 0.13$ & $0.81(0.67-0.95)^{\mathrm{b}}$ & $0.08 \pm 0.08$ & $0.95(0.91-0.99)^{\mathrm{b}}$ & major trans effect \\
\hline
\end{tabular}

Note: For real time PCR data, ratio $(\mathrm{RM} / \mathrm{BY})$ or $(\mathrm{Swap} / \mathrm{BY})=2^{[-\Delta \Delta \mathrm{Ct}]}, \Delta \Delta \mathrm{Ct}=[\Delta \mathrm{Ct}(\mathrm{RM}$ or Swap $)-\Delta \mathrm{Ct}(\mathrm{BY})]$. If ratio $(\mathrm{Swap} / \mathrm{BY})=1$, trans-effect alone; if ratio $(S w a p / B Y) \neq 1$, cis-effect was involved. If ratio $(\mathrm{RM} / \mathrm{BY})=$ ratio (Swap/BY), cis-effect alone; if ratio $(\mathrm{RM} / \mathrm{BY}) \neq$ ratio (Swap/BY), both cis and trans. If the $95 \%$ confidence intervals for RM/BY and Swap/BY have only a minor overlap, we conclude that both cis- and trans-effects were involved. The null hypothesis of Swap/BY $=1$ was tested by the two-tail Student's $t$-test and so was the null hypothesis of RM/BY = Swap/BY. Each reaction was repeated at least three times from different RNA and CDNA samples.

asystematic name.

bignificantly different from $1(P<0.05)$ by $t$-test.

'Significantly different from $1(P<0.01)$ by $t$-test.

Morley et al. 2004; Wittkopp et al. 2004; Hughes et al. 2006; Osada et al. 2006). Results from studies of Drosophila showed that the majority of expression divergence observed both between and within species is due to the variations in the cis-regulatory elements (Wittkopp et al. 2004; Hughes et al. 2006; Osada et al. 2006). The contribution of trans-differences observed between species of fruit flies might not be the original source of expression divergence, but may have accumulated after speciation (Wittkopp et al. 2004). On the other hand, since the yeast data are from different strains in $S$. cerevisiae, the trans variation considered here represents within-species polymorphism.

\section{Methods}

\section{Yeast strains and maintenance}

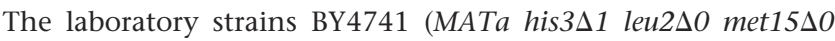
ura3 $\Delta 0$ ) and BY4742 (MAT $\alpha$ his $3 \Delta 1$ leu $2 \Delta 0$ lys $2 \Delta O$ ura3 $\Delta 0)$ are descendants of S288C. The wild strains RM11-1a (MATa leu2 $\Delta O$

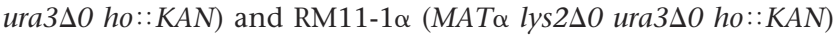
are gifts from Dr. Lee Hartwell (Fred Hutchinson Cancer Research Center, Seattle, WA). They were haploid strains derived from Bb32(3), a natural isolate collected by Robert Mortimer (Mortimer et al. 1994). WL201 is a hybrid strain of BY4741 $\times$ RM11$1 \alpha$, and WL202 is a hybrid strain of BY4742 $\times$ RM11-1a; both were constructed in our lab. Yeast strains were maintained on YPAD.

\section{Growth conditions and extraction of nucleic acids}

Yeast strains were grown in YPAD and harvested at the mid-log phase. Overnight yeast cultures were used to prepare the starting cultures with $\mathrm{OD}_{600}=0.1$ and were grown in YPAD medium at $30^{\circ} \mathrm{C}$ with $250 \mathrm{rpm}$ shaking. The yeast cells were harvested at the $\mathrm{OD}_{600}=1.0$, and the total RNAs were extracted by the hot acid phenol-chloroform method. The mRNA was isolated by using the QIAGEN Oligotex mRNA purification kit (QIAGEN) following the manufacturer's instructions.

\section{Microarray analysis}

Each microarray experiment was conducted with $0.5 \mu \mathrm{g}$ of purified mRNA from each strain. The purified mRNA and oligo dT primers were used for cDNA synthesis with aminoallyl-modified dUTP (Sigma). The reverse transcription was performed following the manufacturer's instructions of the Super-script II kit (Invitrogen). The cDNA samples were cleaned up by Microcon-30 microconcentrators (Millipore) and were labeled with either Cy3 or Cy5 by coupling reactions for microarray experiments. The labeled cDNA samples were purified with the Qiaquick PCR purification kit (QIAGEN) and combined as a cy3/cy5 paired sample. The probes, 40-mer yeast oligos from MWG, were spotted on UltraGAPS coated slides (Corning), and were cross-linked to the slide by UV before use. The hybridization of cDNA samples to the probes was done with the MAUI hybridization System (Biomicro) at $42^{\circ} \mathrm{C}$ for $16 \mathrm{~h}$. The microarray was scanned with GenePix 4000B microarray scanner (Axon Instruments) with the GenePix 5 software package. Each experiment was repeated eight times. Dye-swapping was also performed in each set of experiments to eliminate dye bias (a total of 16 slides). The microarray data obtained from the GenePix 5 were further normalized by the local regression intensity-dependent normalization method (the LOWESS fit), and the scale normalization method.

In order to extract the effect of interest and filter noise resulting from differences in dye-labeling efficiency and variation between/within each array, we apply the two-stage strategy: (1)we use the standard normalization procedure to normalize log ratios of the raw data, and (2) we use an ANOVA model for the normalized $\log$ ratios to estimate the significant levels of the effect of interest.

For the first stage, the composite normalization strategies between/within each array in Yang et al. (2002) can be summarized by the following statistics:

$$
\log _{2} Y_{g r}=\left[\left[\frac{\log _{2} \text { Ratio }_{i g r}-\log _{2} \alpha_{i}(A)}{\beta_{i}}\right]\right]
$$

The term $\log _{2} Y_{g r}$ is the normalized log ratio, which is normalized by the location normalization within array and scale normalization between arrays, Ratio ${ }_{i g r}=R_{i g r} / G_{i g r}$, where $R_{i g r}$ and $G_{i g r}$ represent the raw data of red and green fluorescent intensity in array $i$ for gene $g$ and replicate $r, A=\log _{2} \sqrt{R_{i g r} G_{i g r}}$, the $\log _{2}\left(\alpha_{i}(A)\right)$ term means intensity-dependent normalization, the normalization within array, which can be estimated by the LOWESS fit method, and $\beta_{i}$ is the scale normalization, the normalization between ar-

\section{Genome Research}

www.genome.org 
Table 3. Expression level of TFs (microarray data) and polymorphism rate of the downstream SIM genes

\begin{tabular}{|c|c|c|c|c|c|c|}
\hline TF & $\begin{array}{c}\text { Expression ratio } \\
\text { RM/BY }\end{array}$ & $\begin{array}{l}\text { No. of aa changes } \\
\text { in TF CD }\end{array}$ & Target gene ${ }^{a}$ & $\begin{array}{l}\text { No of } n t \text { changes } \\
\text { in promoter of } \\
\text { target gene }\end{array}$ & $\begin{array}{l}\text { No. of nt changes } \\
\text { in Target } \\
\text { gene CDs }\end{array}$ & Effect \\
\hline CIN5 & 1.020 & 2 & YEL052W & 5 & 12 & both cis and trans \\
\hline CIN5 & 1.020 & 2 & YOR049C & 6 & 7 & both cis and trans \\
\hline GCN4 & 1.011 & 2 & YOL140W & 5 & 8 & both cis and trans \\
\hline HSF1 & 1.093 & 6 & YKL152C & 1 & 1 & both cis and trans \\
\hline INO4 & 1.030 & 0 & YGR157W & 2 & 25 & both cis and trans \\
\hline REB1 & 1.082 & 0 & YOR150W & 12 & 1 & both cis and trans \\
\hline SWI5 & 1.008 & 3 & YPL157W & 4 & 4 & both cis and trans \\
\hline SUM1 & 0.940 & 0 & YJR078W & 4 & 7 & both cis and trans \\
\hline FKH1 & 0.948 & 4 & YFL037W & 2 & 3 & cis alone \\
\hline GCN4 & 1.011 & 2 & YIL117C & 5 & 3 & cis alone \\
\hline HSF1 & 1.093 & 6 & YFL016C & 9 & 8 & cis alone \\
\hline REB1 & 1.082 & 0 & YGL200C & 3 & 1 & cis alone \\
\hline PUT3 & 1.104 & 8 & YOR306C & 11 & 13 & major cis effect \\
\hline YAP7 & 1.031 & 0 & YDL124W & 13 & 9 & major cis effect \\
\hline YAP7 & 1.031 & 0 & YMR318C & 8 & 3 & major cis effect \\
\hline CIN5 & 1.020 & 2 & YAL037W & 0 & 2 & major trans effect \\
\hline FKH1 & 0.948 & 4 & YCR075C & 3 & 3 & major trans effect \\
\hline GCN4 & 1.011 & 2 & YPL264C & 0 & 3 & major trans effect \\
\hline INO2 & 1.021 & 0 & YML075C & 2 & 11 & major trans effect \\
\hline REB1 & 1.082 & 0 & YOL144W & 2 & 14 & major trans effect \\
\hline STB5 & 1.139 & 5 & YDR232W & 6 & 6 & major trans effect \\
\hline SWI6 & 0.973 & 4 & YLR438W & 3 & 4 & major trans effect \\
\hline CBF1 & 0.604 & 2 & YNL094W & 2 & 3 & trans alone \\
\hline DAL82 & 0.928 & 2 & YOL021C & 2 & 2 & trans alone \\
\hline FKH1 & 0.948 & 4 & YDL084W & 0 & 3 & trans alone \\
\hline FKH1 & 0.948 & 4 & YKR054C & 3 & 45 & trans alone \\
\hline GCN4 & 1.011 & 2 & YJL130C & 2 & 17 & trans alone \\
\hline GCN4 & 1.011 & 2 & YNR050C & 4 & 2 & trans alone \\
\hline HSF1 & 1.093 & 6 & YER103W & 6 & 1 & trans alone \\
\hline HSF1 & 1.093 & 6 & YPR154W & 0 & 2 & trans alone \\
\hline REB1 & 1.082 & 0 & YBL050W & 4 & 1 & trans alone \\
\hline REB1 & 1.082 & 0 & YGL198W & 4 & 3 & trans alone \\
\hline REB1 & 1.082 & 0 & YGL248W & 1 & 1 & trans alone \\
\hline REB1 & 1.082 & 0 & YGR241C & 6 & 5 & trans alone \\
\hline REB1 & 1.082 & 0 & YHR146W & 8 & 10 & trans alone \\
\hline REB1 & 1.082 & 0 & YKL072W & 0 & 1 & trans alone \\
\hline REB1 & 1.082 & 0 & YLR223C & 3 & 4 & trans alone \\
\hline REB1 & 1.082 & 0 & YNL113W & 17 & 1 & trans alone \\
\hline REB1 & 1.082 & 0 & YOR326W & 1 & 6 & trans alone \\
\hline SUM1 & 0.940 & 0 & YJR077C & 4 & 3 & trans alone \\
\hline
\end{tabular}

aSystematic name.

rays, which can be estimated by the median absolute deviation $M A D$ (see Yang and Speed 2002; Yang et al. 2002).

For the second stage, we first write down the model and then explain the implication. The ANOVA model for the normalized log ratios is, according to Equation 1 of Landgrebe et al. (2006):

$$
\log _{2} Y_{g r}=\gamma_{R g}-\gamma_{G g}+\tau_{k g}-\tau_{k^{\prime} g}+\varepsilon_{g r}
$$

where $\gamma_{R g}$ and $\gamma_{G g}$ represent the dye-gene interaction term for the dye effect on Cy5 and Cy3 dyes, respectively, $\tau_{k g}-\tau_{k^{\prime} g}$ indicates the term of interest that reflects gene-specific differences in expression for two varieties $\left(k, k^{\prime}\right)$ (like two varieties of BY and RM strains in this paper), and $\varepsilon_{g r}$ denotes the noise. For modeling aspects and applications, see Landgrebe et al. (2006).

The above model can be represented in the matrix form and the corresponding test statistics can be derived for detecting the significant difference in gene expression between BY and RM:

$$
\begin{gathered}
{\underset{\sim}{g}}_{g}=X \underset{\sim}{\beta_{g}}+{\underset{\sim}{\varepsilon_{g}}} \\
t_{g}=\left(\tau_{1 g}-\tau_{2 g}\right) / \sqrt{s_{g}^{2} l^{T}\left(X^{\prime} X\right)^{-1} l},
\end{gathered}
$$

where $Z_{g r}=\log _{2} Y_{g r},{\underset{\sim}{g}}_{g}^{T}=\left[\gamma_{R g} \gamma_{G g} \tau_{1 g} \tau_{2 g}\right], X$ is the corresponding design matrix of the model; $l^{T}=\left(\begin{array}{llll}0 & 0 & 1 & -1\end{array}\right)$, and $s_{g}^{2}$ is the sample variance of $g$. Similar to Landgrebe et al. (2006), we assume that the error is normally distributed with mean 0 and a common variance, so that the test statistics follows a t-distribution with the degree of freedom of $v_{g}=n_{g}$-rank(X), where $n_{g}$ is the number of observations on gene $g$ and $\operatorname{rank}(\mathrm{X})$ is the rank of the design matrix. The corresponding significant level, the $P$-value, can be calculated from the above test statistic for each of the genes under study.

\section{Collection of single TF-regulated genes}

We used a collection of Saccharomyces cerevisiae transcriptional regulators and their target genes predicted from ChIP-chip data (http://jura.wi.mit.edu/young_public/regulatory_code/ GWLD.html) (Harbison et al. 2004). This database includes 203 known TFs and their downstream target genes, from which we collected 1049 genes, each of which is uniquely regulated by only one of 72 TFs at a significance level of $P<10^{-3}$.

\section{Polymorphisms in TFs and promoter regions}

The DNA sequences of the BY4741 strain were downloaded from the Saccharomyces Genome Database (http://www.yeastgenome. 
org/). Sequences for TFs and promoters of their target genes from RM11-1a were determined using an ABI 377A automated DNA sequencer and BigDye sequencing kits (Applied Biosystems) in our lab or obtained from Broad Institute (http://www.broad. mit.edu/annotation/). The DNA sequences of TFs and the promoter regions (intergenic regions) and coding sequences of downstream target genes in both strains were aligned.

\section{Allele quantification with pyrosequencing}

An aliquot of $5 \mu \mathrm{g}$ of total RNA from each strain was used for cDNA synthesis. The reverse transcription was carried out with oligo-dT primers and the Super-script II kit (Invitrogen) following the manufacturer's instructions. After identification of strainspecific nucleotide differences, a 150-200-bp fragment from each gene in two hybrid strains, WL201 and WL202, and in the coculture of parental strains, BY4741 and RM 11-1a, were amplified and sequenced using primer sequences listed in Supplemental Table 5. Pyrosequencing reactions were used to measure the relative abundances of the two alleles in genomic DNA and in cDNA samples from both parental and hybrid pools and were performed according to the manufacturer's instructions (http:// www.pyrosequencing.com/). Pyrosequencing software reports a peak height directly proportional to the number of molecules incorporated into the growing DNA chain. The ratio of allelespecific frequencies $\left(\mathrm{RM}_{\text {parent }} / \mathrm{BY}_{\text {parent }}, \mathrm{RM}_{\text {hybrid }} / \mathrm{BY}_{\text {hybrid }}\right)$, which corresponds to the relative abundances of the BY and RM alleles in the starting sample, was also reported by the pyrosequencing software, PSQ 96MA 2.1.1. The cDNA ratios were normalized with genomic DNA measurements as described in Wittkopp et al. (2004). Because both alleles are extracted and measured in a single sample, this method is insensitive to differences in extraction efficiency and eliminates the need for control genes or quantification of total RNA recovery.

Let $\mathrm{BY}_{\text {hybrid }}$ and $\mathrm{RM}_{\text {hybrid }}$ be the expression levels of the $\mathrm{BY}$ and $\mathrm{RM}$ alleles in the hybrid diploid and let $\mathrm{BY}$ parent and $\mathrm{RM}_{\text {parent }}$ be the expression levels of the BY and RM alleles when the two strains are grown in the same culture. Then, the trans- and ciseffects on the expression differences between $\mathrm{BY}_{\text {parent }}$ and $\mathrm{RM}_{\text {parent }}$ can be judged by the following guidelines:

1. If $\mathrm{RM}_{\text {hybrid }}=\mathrm{BY}$ hybrid $\rightarrow$ trans-effect alone

2. If $\mathrm{RM}_{\text {hybrid }} \neq \mathrm{BY}$ hybrid and

(a) $\mathrm{RM}_{\text {parent }} / \mathrm{BY}_{\text {parent }}=\mathrm{RM}_{\text {hybrid }} / \mathrm{BY}_{\text {hybrid }} \rightarrow$ cis-effect alone

(b) $\mathrm{RM}_{\text {parent }} / \mathrm{BY}_{\text {parent }} \neq \mathrm{RM}_{\text {hybrid }} / \mathrm{BY}_{\text {hybrid }} \rightarrow$ both cis- and trans-effects

The null hypothesis of $\mathrm{RM}_{\text {hybrid }} / \mathrm{BY}_{\text {hybrid }}=1$ was tested by the two-tail Student's $t$-test, and so was the null hypothesis of $\mathrm{RM}_{\text {parent }} / \mathrm{BY}_{\text {parent }}=\mathrm{RM}_{\text {hybrid }} / \mathrm{BY}_{\text {hybrid }}$.

Because the trans-acting effects should be the same in the same genetic background, an equal expression level of the BY and RM alleles in a hybrid diploid indicates differences in transregulation. Therefore, if trans-regulatory divergence completely explains the expression difference of a given gene between the two strains, the allele-specific expression will be approximately the same in the hybrids. However, if the allele-specific expression of a given gene is different in the hybrid, the difference can be attributed to cis-regulatory divergence. If cis-regulatory divergence completely explains the expression difference of a given gene between the two strains, the allele-specific expression ratios of hybrid and parental strains should be approximately equal $\left(\mathrm{RM}_{\text {parent }} / \mathrm{BY}_{\text {parent }}=\mathrm{RM}_{\text {hybrid }} / \mathrm{BY}_{\text {hybrid }}\right)$. If the expression levels of the $\mathrm{BY}$ and $\mathrm{RM}$ alleles in a hybrid are different and the ratio $\mathrm{RM}_{\text {parent }} / \mathrm{BY}_{\text {parent }}$ is different from $\mathrm{RM}_{\text {hybrid }} / \mathrm{BY}_{\text {hybrid }}$, the expres- sion difference between the BY and RM alleles should be due to a combination of cis- and trans-regulatory effects

To further characterize the major effecter in the group of genes affected by both trans- and cis-variations between BY and $\mathrm{RM}$, we divide the distance from the ratio $\mathrm{RM}_{\text {parent }} / \mathrm{BY}_{\text {parent }}$ to 1 into three sections: (1) major trans-effect, if $\mathrm{RM}_{\text {hybrid }} / \mathrm{BY}_{\text {hybrid }}$ is close to 1 ; (2) both trans- and cis-effect, if $\mathrm{RM}_{\text {hybrid }} / \mathrm{BY}_{\text {hybrid }}$ is close to the middle between 1 and $\mathrm{RM}_{\text {parent }} / \mathrm{BY}_{\text {parent }}$; (3) major cis-effect, if $\mathrm{RM}_{\text {hybrid }} / \mathrm{BY}_{\text {hybrid }}$ is close to $\mathrm{RM}_{\text {parent }} / \mathrm{BY}_{\text {parent }}$. In some cases, $\mathrm{RM}_{\text {hybrid }} / \mathrm{BY}_{\text {hybrid }}$ is considerably higher than 1, while $\mathrm{RM}_{\text {parent }} / \mathrm{BY}_{\text {parent }}$ is lower than 1 . In such a case, if the distance from $\mathrm{RM}_{\text {hybrid }} / \mathrm{BY}_{\text {hybrid }}$ to 1 is similar to that from $\mathrm{RM}_{\text {parent }} /$ $\mathrm{BY}_{\text {parent }}$ to 1 , the expression difference is due to both cis- and trans-effect, and if the distance from $\mathrm{RM}_{\text {hybrid }} / \mathrm{BY}_{\text {hybrid }}$ to 1 is substantially larger than from $\mathrm{RM}_{\text {parent }} / \mathrm{BY}_{\text {parent }}$ to 1 , it is major cis-effect.

If the trans-acting factors are the major effecter for the expression divergence between the BY and RM alleles observed in the co-culture conditions, the expression levels of the BY and RM alleles will be approximately equal in a hybrid diploid genetic background, because the trans-acting effects should be the same in the same genetic background. Therefore, if trans-regulatory divergence explains the majority of the expression difference of a given gene between the two strains, the allele-specific expression will be close to that in the hybrids. However, if cis-regulatory divergence explains the majority of the expression difference of a given gene between two strains, the allele-specific expression ratios of hybrid could be close to or higher than the parental strains $\left(\mathrm{RM}_{\text {parent }} / \mathrm{BY}_{\text {parent }} \approx \mathrm{RM}_{\text {hybrid }} / \mathrm{BY}_{\text {hybrid }}\right)$.

\section{Swapping of promoter elements}

The construction was done by PCR-based mutagenesis involving two sequential steps (Gray et al. 2004). The gene of interest was first replaced by an URA3 cassette with $\sim 45$ bp flanking homologous regions to the gene of interest at both ends. The transformation is performed by the LiOAc/SS Carrier DNA/PEG method (Gietz and Woods 2002) and transformants were selected as $\mathrm{Ura}^{+}$ colonies. The insertion of URA3 at the targeted site was confirmed by diagnostic PCR and sequencing. The inserted URA3 was further replaced by a second transformation with the appropriate fragment into the URA3-inserted strain. The transformants were selected by 5-FOA counter selection. Only the strains that carried the desired sequence will survive and form colonies on the medium with 5-FOA $(1 \mu \mathrm{g} / \mathrm{mL})$. The constructions at the targeted site were confirmed by diagnostic PCR and sequencing.

\section{Expression shift examined by real-time PCR}

An aliquot of $0.5 \mu \mathrm{g}$ of purified mRNA from each strain was used for cDNA synthesis and the reverse transcription was carried out with oligo-dT or random hexamer primers following the manufacture's instructions of the Super-script II kit (Invitrogen). Realtime PCR analyses were carried out in a final volume of $25 \mu \mathrm{L}$ that contained $2 \mu \mathrm{L}$ of diluted cDNA $(1 \mathrm{ng} / \mu \mathrm{L}), 1 \mu \mathrm{L}$ each of gene-specific forward and reverse primers $(5 \mu \mathrm{M})$, and $12.5 \mu \mathrm{L}$ of SYBR Green Supermix from Bio-Rad with 40 cycles of $95^{\circ} \mathrm{C}$ for 15 sec and $60^{\circ} \mathrm{C}$ for $1 \mathrm{~min}$. The primers were designed by using the Primer Express software from Applied Biosystems. The relative expression of each gene was normalized to that of the Act1 gene $(\Delta \mathrm{Ct})$ and quantified with the $\Delta \Delta \mathrm{Ct}$ relative quantification method and the relative expression ratio was calculated following ABI's suggestion, ratio $(\mathrm{RM} / \mathrm{BY})=2^{[-\Delta \Delta \mathrm{Ct}]}$. The amplification efficiency of each primer pair was tested by using twofold serial dilutions of the templates as suggested by Applied Biosystems and the amplification efficiency of the target gene and the refer-

\section{Genome Research}

www.genome.org 
ence gene were approximately equal. The influence of cis- or trans-effect was determined by the same guideline as used for analyzing the pyrosequencing data.

1. if ratio Swap/BY $=1 \rightarrow$ trans-effect

2. if ratio Swap/BY $\neq 1$ and

(a) if ratio $\mathrm{RM} / \mathrm{BY}=$ ratio Swap/BY $\rightarrow$ cis-effect alone

(b) if ratio $\mathrm{RM} / \mathrm{BY} \neq$ ratio (Swap/BY) $\rightarrow$ both cis- and trans-effect

The null hypothesis of Swap/BY = 1 was tested by the two-tail Student's $t$-test, and so was the null hypothesis of $\mathrm{RM} / \mathrm{BY}=$ Swap/BY.

\section{Primers}

Primers used for gene disruption, promoter swapping, and allele quantification are listed in Supplemental Table 5.

\section{Acknowledgments}

We thank Josh Rest, Henry Lu, P.J. Wittkopp, and Z. Luo and the reviewers for valuable comments. This study was supported by Academia Sinica and NIH grants to W.H.L.

\section{References}

Brem, R.B., Yvert, G., Clinton, R., and Kruglyak, L. 2002. Genetic dissection of transcriptional regulation in budding yeast. Science 296: 752-755.

Bush, R.M. and Paigen, K. 1992. Evolution of $\beta$-glucuronidase regulation in the genus Mus. Evolution Int. J. Org. Evolution 46: 1-15.

Carroll, S.B. 2000. Endless forms: The evolution of gene regulation and morphological diversity. Cell 101: 577-580.

Carroll, S.B., Grenier, J.K., and Weatherbee, S.D. 2001. From DNA to diversity: Molecular genetics and the evolution of animal design. Blackwell Science, Malden MA.

Cowles, C.R., Hirschhorn, J.N., Altshuler, D., and Lander, E.S. 2002 Detection of regulatory variation in mouse genes. Nat. Genet. 32: 432-437.

Daborn, P.J., Yen, J.L., Bogwitz, M.R., Le Goff, G., Feil, E., Jeffers, S. Tijet, N., Perry, T., Heckel, D., Batterham, P., et al. 2002. A single p450 allele associated with insecticide resistance in Drosophila. Science 297: 2253-2256.

DeRisi, J.L., Iyer, V.R., and Brown, P.O. 1997. Exploring the metabolic and genetic control of gene expression on a genomic scale. Science 278: $680-686$.

Forsburg, S.L. and Guarente, L. 1989. Identification and characterization of HAP4: A third component of the CCAAT-bound HAP2/HAP3 heteromer. Genes \& Dev. 3: 1166-1178.

Gerhart, J. and Kirschner, M. 1997. Cell, embryos, and evolution: Toward a cellular and developmental understanding of phenotypic variation and evolutionary adaptability. Blackwell Science, Malden, MA.

Gietz, R.D. and Woods, R.A. 2002. Transformation of yeast by lithium acetate/single-stranded carrier DNA/polyethylene glycol method. In Methods in enzymology (eds. C. Guthrie and G.R. Fink), pp. 87-96. Academic Press, San Diego, CA.

Gray, M., Kupiec, M., and Honigberg, S.M. 2004. Site-specific genomic (SSG) and random domain-localized (RDL) mutagenesis in yeast. BMC Biotechnol. 4: 7. doi: 10.1186/1472-6750-4-7.

Gu, Z., David, L., Petrov, D., Jones, T., Davis, R.W., and Steinmetz, L.M. 2005. Elevated evolutionary rates in the laboratory strain of Saccharomyces cerevisiae. Proc. Natl. Acad. Sci. 102: 1092-1097.

Harbison, C.T., Gordon, D.B., Lee, T.I., Rinaldi, N.J., Macisaac, K.D., Danford, T.W., Hannett, N.M., Tagne, J.B., Reynolds, D.B., Yoo, J., et al. 2004. Transcriptional regulatory code of a eukaryotic genome. Nature 431: 99-104.
Hughes, K.A., Ayroles, J.F., Reedy, M.M., Drnevich, J.M., Rowe, K.C., Ruedi, E.A., Caceres, C.E., and Paige, K.N. 2006. Segregating variation in the transcriptome: cis regulation and additivity of effects. Genetics 173: 1347-1355.

Landgrebe, J., Bretz, F., and Brunner, E. 2006. Efficient design and analysis of two colour factorial microarray experiments. Comput. Stat. Data Anal. 50: 499-517.

Laurie-Ahlberg, C.C. and Bewley, G.C. 1983. Naturally occurring genetic variation affecting the expression of sn-glycerol-3-phosphate dehydrogenase in Drosophila melanogaster. Biochem. Genet. 21: 943-961.

Lee, T.I., Rinaldi, N.J., Robert, F., Odom, D.T., Bar-Joseph, Z., Gerber, G.K., Hannett, N.M., Harbison, C.T., Thompson, C.M., Simon, I., et al. 2002. Transcriptional regulatory networks in Saccharomyces cerevisiae. Science 298: 799-804.

Li, W.H. 1997. Molecular evolution. Sinauer Associated, Inc., Sunderland, MA.

Morley, M., Molony, C.M., Weber, T.M., Devlin, J.L., Ewens, K.G., Spielman, R.S., and Cheung, V.G. 2004. Genetic analysis of genome-wide variation in human gene expression. Nature 430: $743-747$.

Mortimer, R.K., Romano, P., Suzzi, G., and Polsinelli, M. 1994. Genome renewal: A new phenomenon revealed from a genetic study of 43 strains of Saccharomyces cerevisiae derived from natural fermentation of grape musts. Yeast 10: $1543-1552$.

Osada, N., Kohn, M.H., and Wu, C.I. 2006. Genomic inferences of the cis-regulatory nucleotide polymorphisms underlying gene expression differences between Drosophila melanogaster mating races. Mol. Biol. Evol. 23: 1585-1591.

Purugganan, M.D. 2000. The molecular population genetics of regulatory genes. Mol. Ecol. 9: 1451-1461.

Rifkin, S.A., Kim, J., and White, K.P. 2003. Evolution of gene expression in the Drosophila melanogaster subgroup. Nat. Genet. 33: 138-144.

Rockman, M.V. and Wray, G.A. 2002. Abundant raw material for cis-regulatory evolution in humans. Mol. Biol. Evol. 19: 1991-2004.

Rockman, M.V. and Kruglyak, L. 2006. Genetics of global gene expression. Nat. Rev. Genet. 7: 862-872.

Ronald, J., Brem, R.B., Whittle, J., and Kruglyak, L. 2005. Local regulatory variation in Saccharomyces cerevisiae. PLoS Genet. 1: e25. doi: 10.1371/journal.pgen.0010025.

Schadt, E.E., Monks, S.A., Drake, T.A., Lusis, A.J., Che, N., Colinayo, V., Ruff, T.G., Milligan, S.B., Lamb, J.R., Cavet, G., et al. 2003. Genetics of gene expression surveyed in maize, mouse and man. Nature 422: 297-302.

Stern, D.L. 2000. Evolutionary developmental biology and the problem of variation. Evolution Int. J. Org. Evolution 54: 1079-1091.

Streelman, J.T. and Kocher, T.D. 2002. Microsatellite variation associated with prolactin expression and growth of salt-challenged tilapia. Physiol. Genomics 9: 1-4.

Tautz, D. 2000. Evolution of transcriptional regulation. Curr. Opin. Genet. Dev. 10: $575-579$.

Wittkopp, P.J., Haerum, B.K., and Clark, A.G. 2004. Evolutionary changes in cis and trans gene regulation. Nature 430: $85-88$.

Wray, G.A., Hahn, M.W., Abouheif, E., Balhoff, J.P., Pizer, M., Rockman, M.V., and Romano, L.A. 2003. The evolution of transcriptional regulation in eukaryotes. Mol. Biol. Evol. 20: 1377-1419.

Yang, Y.H. and Speed, T. 2002. Design issues for cDNA microarray experiments. Nat. Rev. Genet. 3: 579-588.

Yang, Y.H., Dudoit, S., Luu, P., Lin, D.M., Peng, V., Ngai, J., and Speed, T.P. 2002. Normalization for cDNA microarray data: A robust composite method addressing single and multiple slide systematic variation. Nucleic Acids Res. 30: e15.

Yvert, G., Brem, R.B., Whittle, J., Akey, J.M., Foss, E., Smith, E.N., Mackelprang, R., and Kruglyak, L. 2003. Trans-acting regulatory variation in Saccharomyces cerevisiae and the role of transcription factors. Nat. Genet. 35: 57-64.

Received January 25, 2007; accepted in revised form April 23, 2007. 


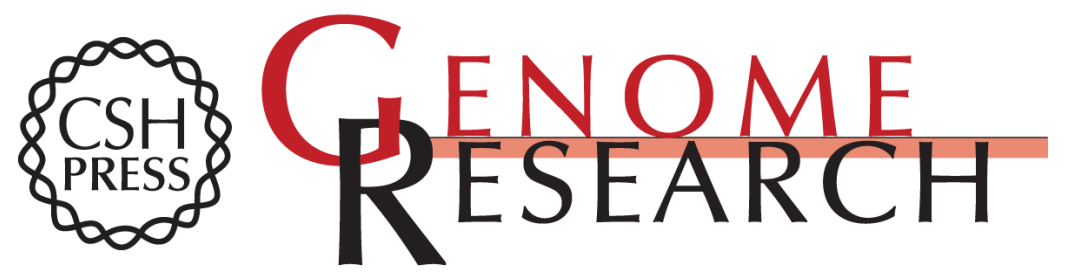

\section{Expression evolution in yeast genes of single-input modules is mainly due to changes in trans-acting factors}

Daryi Wang, Huang-Mo Sung, Tzi-Yuan Wang, et al.

Genome Res. 2007 17: 1161-1169 originally published online July 5, 2007 Access the most recent version at doi:10.1101/gr.6328907

Supplemental Material

References License

Email Alerting Service
http://genome.cshlp.org/content/suppl/2007/07/06/gr.6328907.DC1

This article cites 31 articles, 7 of which can be accessed free at: http://genome.cshlp.org/content/17/8/1161.full.html\#ref-list-1

Receive free email alerts when new articles cite this article - sign up in the box at the top right corner of the article or click here.

\section{Affordable, Accurate Sequencing.}

